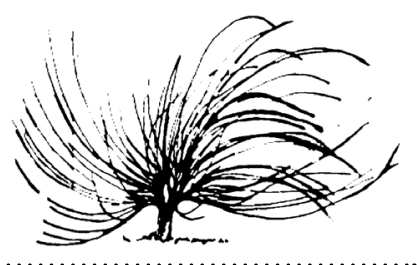

\title{
Experiencia educativa focalizada en el personal docente para promover el compromiso escolar. Percepciones de estudiantes
}

\author{
Daiana Yamila Rigo ${ }^{1}$ \\ Universidad Nacional de Río Cuarto \\ Argentina \\ daianarigo@hotmail.com
}

\begin{abstract}
Resumen
El escrito describe una investigación realizada en el nivel primario de educación, basada en los postulados teóricos de la teoría de la autodeterminación y compromiso escolar con los objetivos de: (a) examinar la configuraciones naturales de apoyos a la autonomía y estructura ofrecida por docentes y percibida por sus estudiantes, (b) describir la asociación entre dichas percepciones con las autovaloraciones estudiantiles en cada dimensión de compromiso, (c) diseñar una experiencia educativa tendiente a promover los apoyos brindados por docentes en las clases y sobre las tareas escolares, y (d) valorar pos experiencia educativa la asociación entre las percepciones de apoyo y el compromiso autovalorado por el estudiantado. La muestra estuvo conformada por escolares de $5{ }^{\text {to }}$ grado
\end{abstract}

Recibido: 19 de octubre de 2019. Aprobado: 2 de setiembre de 2020 .

http://dx.doi.org/10.15359/rep.16-1.10

1 Doctora en Psicología por la Facultad de Psicología, UNSL. Máster en Ciencias Sociales, UNRC. Máster en Psicología de la Educación, UMU, España. Licenciada en Psicopedagogía, UNRC. Profesora de grado y postgrado en la UNRC-UNC. Investigadora asistente del CONICET. Ha realizado y realiza tarea de investigación y docencia en relación con CONICET, AECI, FONCyT, SECyt-UNRC. Dirige, codirige y colaboradora en diversos proyectos de investigación orientados a la mejora educativa. Directora de trabajos finales de grado y postgrado. Sus trabajos de investigación refieren al campo de la educación, el diseño instructivo y el compromiso. Autora de artículos para libros y revistas sobre temas de su especialidad. https://orcid.org/0000-0003-0312-6429 
$(\mathrm{n}=53)$, de una escuela pública de Argentina. La recolección de datos se llevó a cabo a partir de la administración de un cuestionario ad hoc al finalizar la experiencia; asimismo, se usaron dos instrumentos estandarizados antes y después de la investigación, para valorar la percepción de los apoyos recibidos por la parte docente y el compromiso del estudiantado. Los resultados muestran que el alumnado logra percibirse más involucrado con sus aprendizajes cuando aprecia que las tareas escolares se definen con una instrucción que apoya sus iniciativas, pero a la vez ofrece una adecuada definición de las metas y objetivos de aprendizaje. Lo encontrado ofrece lineamientos concretos para promover prácticas educativas tendientes a socavar los comportamientos de desvinculación de la mano de un estilo docente que apoye al estudiantado hacia la toma de decisiones y con retroalimentaciones formativas.

Palabras clave: Compromiso, autonomía, retroalimentación, experiencia educativa.

\begin{abstract}
This paper describes an investigation carried out at the primary level of education based on the theoretical postulates of the theory of self-determination and school engagement with the objectives of: (a) examining the natural configurations of support for autonomy and reference structure by teachers and perceived by the students, (b) describing the association between different perceptions with the students' self-assessments in each dimension of engagement, (c) designing an educational experience aimed at promoting the support provided by teachers in class and on homework school children, and (d) assessing the association between perceptions of support and self-valued engagement by students after educational experience. The sample was composed up of 5 th grade students $(n=53)$, from a public school of Argentina. Data collection was carried out based on the administration of an ad hoc questionnaire at the end of the experience. In addition, two standardized instruments were used before and after the investigation to assess the perception of the support received by the teacher and the
\end{abstract}


students' engagement. The results show that students get more involved with their learning when they appreciate that school tasks are defined under an instruction that supports their initiatives, but at the same time, offers an adequate definition of learning goals and objectives. The findings offer concrete guidelines to scaffold educational practices aimed at undermining the disengagement behaviors in the hands of a teaching style that supports students towards decision making and with formative feedback.

Keywords: engagement, autonomy, feedback, educational experience

\section{Introducción}

nivel internacional y nacional, un número significativo
de estudiantes de nivel primario de educación presenta
comportamientos en el aula asociados a procesos de desvinculación, tales como falta de interés, ausentismo, escaso grado de participación, entre otros, lo cual resulta en consecuencias para el aprendizaje, consecución y finalización de la escuela (CAF, 2018). Específicamente, en Argentina, algunas problemáticas se visualizan en los últimos resultados de la prueba Aprender, donde el $33 \%$ de docentes señala que el ausentismo de sus estudiantes representa un problema en la institución (Aprender, 2018).

Las recomendaciones brindadas por el informe sobre calidad educativa TERCE - Tercer Estudio Regional Comparativo y Explicativo- indican que mejorar las motivaciones estudiantiles se asocia a la calidad de los apoyos y, entre ellos, se destacan los ofrecidos por el personal docente (UNESCO 2015). Entendemos, por tanto, que el ambiente instruccional es un poderoso vehículo para propiciar la implicación de estudiantes en el aula y hacia las tareas escolares. Al respecto, tanto el modelo contextual de Lam, Wong Yang y Liu (2012), como la complejidad ambiental de Shernoff, et al. (2016), muestran evidencia de que el entorno y clima de la clase, junto al diseño instructivo, son claves para pensar en el compromiso de estudiantes.

Concretamente, el último informe ejecutivo de TERCE realizado por la UNESCO (2015) expone que la calidad de los aprendizajes, 
si bien se asocia a los recursos del aula, estos no son suficientes para asegurar un proceso educativo adecuado, puesto que su uso debe estar soportado por las interacciones del aula que promuevan la comprensión, la participación y el interés estudiantiles. Aportes que han sido puntualizados por estudios más recientes en el marco de la teoría de la autodeterminación -TAD-, referidos a dimensiones del estilo docente que podrían contribuir a mejorar la implicación del alumnado con sus aprendizajes, enfatizan los apoyos dirigidos a soportar la autonomía y ofrecer una clara estructura de trabajo.

Sobre tales contribuciones, el presente estudio contempla las dimensiones definidas por la TAD en la puesta en marcha de una experiencia educativa, para explorar las implicancias que tienen sobre el compromiso del estudiantado. Específicamente, la TAD desarrollada en el contexto educativo aporta lineamientos generales para definir el contexto instructivo, en pos de promover el compromiso de estudiantes. Entiende que el alumnado presenta tres necesidades motivacionales, tales como, la autonomía, la competencia y la relación:

La autonomía se satisface cuando el estudiante siente que toma opciones y se motiva por factores intrínsecos más que externos, la competencia se favorece cuando la estructura de la clase permite alcanzar los resultados deseados y la relación ocurre cuando profesores y pares crean un entorno de apoyo y preocupación. (Albornoz, Villablanca y Díaz, 2019, pp. 83)

Se supone que el estilo docente en la configuración de los ambientes y actividades de aprendizaje que contemplen esas necesidades y brinden oportunidades para satisfacerlas, conducirá a mayores niveles de compromiso del estudiantado con las tareas escolares. Partiendo de ese supuesto, diseñamos una experiencia educativa con el objetivo general de comprender la percepción que tiene la población estudiantil sobre los apoyos ofrecidos por sus docentes en materia de autonomía y estructura y su compromiso hacia las tareas de aprendizaje que les asignan.

Adoptamos la postura de Dubet y Martuccelli (2000), para comprender que las experiencias educativas son producciones sociales conjuntas, compuestas de propósitos múltiples y a menudo contradictorios, en las cuales los intereses de los sujetos convergen en un equilibrio medianamente estable, lo que da lugar a que las percepciones sobre los 
apoyos percibidos y el compromiso adoptado sean una construcción situada, individual, pero colectiva, a la vez, en torno a las vivencias de la clase.

Entendemos que las percepciones de estudiantes sobre el entorno de aprendizaje, así como, de los apoyos brindados por sus docentes y el compromiso autoinvolucrado, son el resultado de la interacción entre los comportamientos individuales y las influencias del contexto educativo (Könings, 2007; Könings, Brand-Gruwel y van Merriënboer, 2005). Es decir, el estudiantado le da significado a los contextos de enseñanza y aprendizaje a partir de las representaciones que va formando sobre las tareas desarrolladas durante la clase y los intercambios establecidos con sus docentes. Könings (2007) considera que conocer estas percepciones desarrolladas con respecto a los contextos educativos es crucial para generar rupturas y cambios en los diseños instructivos, en tanto su desconocimiento contribuye a la desvinculación con la escuela. Asimismo, postula que juegan un papel prominente para el rediseño de los contextos de aprendizaje y la generación de nuevas percepciones que conduzcan a mejores aprendizajes.

En tal sentido, la presente investigación parte de conocer cómo los grupos de estudiantes de nivel primario de educación se perciben comprometidos y su vínculo con la percepción sobre los apoyos percibidos por los maestros y maestras respecto a la autonomía y la estructura, para luego generar una experiencia educativa que tienda a promover aquellos aspectos en torno al estilo docente que potencien el compromiso estudiantil. Entendemos que las percepciones del estudiantado sobre un entorno de aprendizaje están relacionadas con sus manifestaciones afectivas, conductuales, cognitivas y agénticas.

En ese marco, la experiencia educativa desarrollada se inscribe en una investigación descriptiva con estudiantes del nivel primario de educación y habilita un espacio de análisis vinculado a explorar las percepciones que construyen sobre un entorno micro, como es el aula, definido en las interacciones que allí tienen lugar, entre estudiantes y docentes (Santana-Armas, Noda-Rodríguez y Pérez-Sánchez, 2018). Específicamente, interesa describir cómo la modificación del contexto instructivo, sustentada en un estilo docente orientado a promover la autonomía con base en una estructura clara de trabajo áulico, se relaciona con las percepciones que el estudiantado elabora sobre los 
apoyos recibidos y el compromiso reportado hacia la resolución de las tareas escolares.

\section{Desarrollo conceptual}

Fredricks, Reschly y Christenson (2019) entienden que el compromiso es una metaconstrucción que unifica diferentes aportes teóricos y puede proporcionar una imagen más rica de cómo el alumnado piensa, actúa y se siente en la escuela. Consiste en cuatro dimensiones distintas, pero interrelacionadas -afectiva, conductual, cognitiva y agéntica- que se moldean en función de las características áulicas y el estilo docente (Appleton, Cristenson, Kin y Reschly, 2006; Fredricks, Blumenfeld y Paris, 2004; Reeve, 2013; Rigo, 2017; Rigo y Donolo, 2018).

Desde la perspectiva del entorno más la persona, Eccles y Midgley (1989) y Eccles, Lord y Midgley (1991) suponen que el compromiso se verá influenciado por el ajuste entre las necesidades y objetivos de una persona y las oportunidades disponibles para satisfacer las necesidades y metas en su entorno. La teoría, con grandes contribuciones desde su formulación, aporta evidencia para explicar la desmotivación y desvinculación de estudiantes durante los años de escolarización, como producto de un desajuste entre sus necesidades de mayor autonomía y oportunidades para explorar sus intereses y el contexto instruccional que muestra una mayor tendencia hacia el control y la disciplina. Asimismo, el modelo de autosistema de desarrollo motivacional de Connell y Wellborn (1991), basado en la TAD, vincula también los factores contextuales, como la estructura del aula, el apoyo a la autonomía y la participación, con patrones de compromiso que el alumnado elabora en función de la satisfacción de sus necesidades básicas de competencia, relación y autonomía en contextos particulares.

Reeve, Cheon y Jang (2019), en una contribución reciente, entienden que el compromiso refiere a la participación activa en una actividad de aprendizaje, dirigida por objetivos y propósitos específicos, entre los cuales se mencionan, conseguir mejores logros educativos, satisfacer sus motivaciones y crear entornos de aprendizaje favorables $\mathrm{y}$ afines a sus intereses.

Esta definición no solo recupera la idea del compromiso como un meta-constructo integrado por tres dimensiones -afectiva, cognitiva y conductual-, sino que además incorpora la agencia como la cuarta faceta proactiva del término. Rescata, asimismo, la interacción 
del sujeto con el entorno, mediada por metas educativas que moldean el compromiso.

A la importancia del contexto áulico y escolar como elemento central, se suman las intenciones y acciones que el alumnado imprime sobre el entorno para crear situaciones educativas que promuevan sus intereses. El compromiso, desde esta óptica, no depende tan solo de cómo se configure el contexto de la clase para captar la atención, aumentar la participación o lograr un estudiantado más autorregulado, sino que además se empieza a pensar en estudiantes como sujetos activos de los procesos de vinculación, quienes dirigen sus acciones y comportamientos para modificar el entorno en función de sus necesidades motivacionales, en tanto ese entorno habilite espacios para que logren expresar sus agencias en las aulas (Appleton, Cristenson, Kin y Reschly, 2006; Fredricks, Blumenfeld y Paris, 2004; Reeve, 2013).

Lo importante del compromiso agente es comprender que la definición de contexto de aula es producto de una interacción espiralada, no unidireccional del personal docente hacia el estudiantado, sino también a la inversa. Pensar la implicación desde esta nueva perspectiva supone ampliar los marcos de referencia a partir de los cuales se pueda pensar en experiencias educativas tendientes a promover la percepción que estudiantes construyen sobre sus compromisos escolares, en tanto reconozcan andamios orientados a su promoción por parte de sus docentes. Al respecto, Deci y Ryan (2008) entienden que los sujetos estarán más o menos interesados en una actividad, en la medida en que experimenten y aprecien la satisfacción de sus necesidades al involucrarse en ella.

Desde la TAD, Reeve (2016) postula que tal satisfacción se relaciona con el estilo motivacional que adopta cada docente, como el tono interpersonal y el comportamiento cara a cara que los maestros y maestras utilizan habitualmente para involucrar a sus estudiantes en las actividades de aprendizaje que proporcionan. Reeve, Cheon y Jang (2019) puntualizan en dos dimensiones: la definición de una clara estructura de trabajo áulico y los apoyos al promover la autonomía estudiantil, y reconocen que la estructura necesariamente debe ofrecerse como andamio de la autonomía otorgada. Sierens, Vansteenkiste, Goossens, Soenens y Dochy, (2009), Van Loon, Ros y Martens (2012), Stroet, Opdenakker y Minnaert (2013), Mouratidis, Michou, Aelterman, Haerens y Vansteenkiste (2017) y Schenke (2018) muestran que 
combinar ambas dimensiones podría contribuir a incrementar la motivación y el aprendizaje.

Un entorno instructivo con apoyos hacia la autonomía es un contexto en el que la presión externa es mínima, se reconocen las metas personales de estudiantes y se les ofrecen opciones para poder elegir, acciones que contribuyan a sentir menos control sobre sus decisiones y comportamientos (Deci y Ryan, 2008). Asimismo, el papel docente consiste en proporcionar orientaciones con un lenguaje no directivo que les aliente a tomar iniciativas. Ryan y Grolnick (1986) y Sarrazin, Tessier y Trouilloud (2006), precisan que tales ambientes estimulan la curiosidad estudiantil y les alienten a tomar desafíos.

Jang, Reeve y Deci (2010), Oga-Baldwin y Nakata (2015), Hospel y Galand (2016) y Reeve, Cheon y Jang (2019) puntualizan que un ambiente que solo ofrece autonomía, puede generar mayores distracciones y abrumar con demasiadas alternativas; con lo cual la estructura también juega un papel importante. Proporcionarla hace que el ambiente de aprendizaje sea menos confuso y más consistente y predecible para estudiantes. Significa brindar ayuda, apoyo, estrategias apropiadas y orientaciones para que el estudiantado realice una tarea con éxito. Además, supone explicitar las metas y exceptivas con claridad y andamiar hacia su consecución ofreciendo procedimientos claros para lograrlas. Supone, también, entregar retroalimentaciones que apoyen la reflexionar sobre lo realizado.

Stroet, Opdenakker y Minnaert (2013) sintetizan varios componentes que integran a una $\mathrm{u}$ otra dimensión del estilo docente. Respecto a autonomía, plantean que una enseñanza basada en opciones, relevancia y respeto genera un contexto de aprendizaje donde sus estudiantes tienen mayores posibilidades de ser agentes causales, experimentar volición y actuar de acuerdo con su sentido de identidad. En relación con la estructura, los maestros y maestras pueden proporcionarla por medio de cuatro rasgos presentes en las clases y las tareas escolares planificadas, tales como, claridad, orientaciones, apoyos con base en la clarificación de expectativas sobre la tarea escolar y retroalimentaciones informativas.

Investigaciones previas formulan que ambos aspectos se pueden promover a partir de diversos recursos. Arias y Vega (2016) afirman que las tecnologías de información y comunicación (TIC) se han convertido en poderosas herramientas que, al ser integradas al contexto educativo, 
permiten ir adquiriendo autonomía en los aprendizajes; asimismo, Llorent y Torres-Porras (2018) proponen las visitas escolares, o itinerarios formativos en donde el estudiantado puede poner en práctica in situ conocimientos de las asignaturas que esté cursando, como un verdadero espacio de aprendizaje ligado a las experiencias laborales futuras, y como medio para fortalecer la utilidad y la aplicación del conocimiento teórico. Por último, Jiménez-Jiménez, Sosa-Alonso, Santana-Bonilla y Hernández-Rivero (2018) exponen que el uso planificado de retroalimentaciones formativas, posibilita al estudiantado prever, al principio de la asignatura, el objeto de valoración, mantenerlo durante la resolución de las tareas y reflexión final.

Desde estos postulados, y asumiendo que el compromiso es maleable, y en la línea de la meta formulada por Fredricks et al. (2019), acerca de la importancia de generar instancias educativas tendientes a promover el compromiso no solo de aquel estudiantado que corre el riesgo de abandonar la escuela, sino también de quienes permanecen, pero se aburren y desinteresan, encontramos necesario traducir los desarrollos teóricos en implicancias prácticas, en el marco de una experiencia educativa en el nivel primario de educación, con el objetivo general de propiciar un entorno educativo donde los grupos de estudiantes que perciban los apoyos que el personal docente dirige hacia una mayor autonomía y una clara estructura de trabajo áulico, también desarrollen una valoración aún mayor del compromiso reportado.

\section{Metodología}

El estudio desarrollado se inscribe en una investigación descriptiva con diseño cuantitativo (Sampieri, Collado y Lucio, 2010), que busca examinar la configuraciones naturales de apoyos a la autonomía y estructura ofrecida por docentes y percibida por sus estudiantes, describir la asociación entre dichas percepciones con las autovaloraciones estudiantiles en cada dimensión de compromiso escolar, diseñar una experiencia educativa tendiente a promover los apoyos brindados por docentes durante las clases y la realización de las tareas escolares, y valorar la asociación tras la experiencia educativa entre las percepciones de apoyo y el compromiso autovalorado por el alumnado. 


\section{Muestra}

La muestra estuvo integrada por 53 estudiantes de 5 to $^{\text {grado, de }}$ una escuela pública, nivel primario, de la provincia de Córdoba, departamento Río Cuarto, Argentina. Estuvo conformada por escolares de las 2 divisiones de turno mañana, de una edad media de 10,21 años $(\mathrm{S}=0,41)$. Se destaca que se contó con el asentimiento informado de los padres y madres de familia.

\section{Instrumentos}

Para valorar el compromiso de estudiantes se utilizó una adaptación y actualización de la "Escala de compromiso hacia las tareas escolares en las clases del nivel primario de educación", desarrollada por Rigo y Donolo (2018), la cual incluye la dimensión agéntica y presenta una adecuada estructura interna e índice de fiabilidad $\left(\mathrm{X}^{2}=\right.$ $296.12, \mathrm{p}=.001, \mathrm{X}^{2} / \mathrm{gl}=1.3, \mathrm{CFI}=.80, \mathrm{GFI}=.86, \mathrm{RMSE}=.059-\mathrm{IC}$ $90 \%=.039 / .076-, \mathrm{a}=.83$ ), además de la afectiva, conductual y cognitiva; y a partir de una escala Likert de 5 puntos cada sujeto valoró cada enunciado ( 1 = Casi nunca; 5 = Siempre). Para los ítems de la dimensión agéntica, se tomó en consideración los aportes de Veiga (2013).

Asimismo, se consultó al alumnado sobre la percepción del aula, antes y después de la investigación, a través del cuestionario sobre el contexto social de la clase, para indagar criterios relacionados con el estilo docente, a partir de las dimensiones apoyo a la autonomía (tales como, control decido, opciones ofrecidas, respeto hacia la idea de estudiantes y relevancia de las actividades) y claridad en la estructura (comunicación de expectativas, monitoreo y modelado cognitivo, y comportamiento predecible). La versión usada se adaptó de Belmont, Skinner, Wellborn y Connel (1992) y se contemplaron solo 16 ítems referidos a las dimensiones valoradas, cuyo formato de respuesta se distribuye en una escala Likert de 4 puntos ( 1 = Para nada cierto; $4=$ Muy cierta). Este mismo se adaptó contemplando las pautas elaboradas por Zapata y Canet (2008), y presentó una adecuada consistencia interna, $=.89$.

Finalmente, se administró un cuestionario ad hoc para conocer en detalle los aspectos favorecidos a partir de la experiencia educativa. Algunas de las preguntas incluidas en estas dos últimas instancias estuvieron orientadas a conocer, si consideraron fructífero que las maestras informaran sobre sus desempeños en la clase y en las tareas, 
sobre las dificultades encontradas y los recursos -físicos (Internet) y sociales (visita de personas expertas)- utilizados para resolverlas, sobre las percepciones en torno a los espacios para expresar sus ideas o proponer formas de trabajar, así como sobre, si las orientaciones ofrecidas durante las tareas escolares fueron claras, comprensibles, flexibles y constructivas y, acerca de cómo actuaron cuando no sabían qué hacer o estaban desorientados en la tarea.

\section{Procedimiento}

Cada estudiante de $5 .^{\text {to }}$ grado completó el cuestionario para autovalorar su compromiso con las tareas escolares y la escala para describir su percepción sobre el contexto social de la clase antes de la experiencia educativa, con base en dos dimensiones: autonomía y estructura.

Como parte de la experiencia educativa, se desarrollaron varias instancias de intercambio con la docente de grado, sobre la conceptualización teórica acerca de qué implica ofrecer andamios para promover la autonomía en estudiantes según una clara estructura de trabajo áulico. Se incluyeron dos momentos:

1. Lectura y aprendizaje teórico: durante seis encuentros con la docente se abordaron conceptos centrales de la TAD relacionados con el estilo docente tendientes a promover en el alumnado la toma de decisiones y explicitar las expectativas de trabajo, concretamente se profundizó sobre los aspectos delimitados por Jang, Reeve y Deci (2010), sintetizados en las Tablas 1 y 2. Se analizó cada dimensión en relación con la educación, el compromiso de estudiantes, los procesos de enseñanza y aprendizaje y la transferencia hacia las tareas escolares. 


\section{Tabla 1}

Apoyos orientados a promover la autonomía

\section{El personal docente...}

-Crea oportunidades para la iniciativa, interés, disfrute y desafío.

-Proporciona opciones. Identifica el valor, el significado y la importancia de las tareas. Usa un lenguaje informativo y flexible.

-Reconoce y acepta las expresiones negativas de sus estudiantes

-Escucha atentamente y comprensivamente. Los comportamientos negativos son contemplados.

Nota: elaboración propia.

\section{Tabla 2}

Apoyos orientados a ofrecer estructura

\section{El personal docente...}

-Al inicio de la clase, ofrece consignas claras, comprensibles, explícitas y detalladas. Tareas bien organizadas.

-Durante la tarea, da orientaciones claras y supervisadas, con un plan de acción y metas de aprendizajes explicitas.

-Durante la retroalimentación, brinda información constructiva y relevante para orientar la tarea.

Nota: elaboración propia.

2. Planificación e implementación de la experiencia educativa: durante un semestre y medio, en dos horas semanales, la maestra planificó las tareas escolares y orquestó la clase teniendo en cuenta los aspectos sintetizados en las Tablas 1 y 2, se lo ayudó a ofrecer un marco de trabajo áulico más autónomo y claro en información. Los intercambios tomaron particularidades afines a las circunstancias y características del aula y sus estudiantes, los contenidos y las actividades propuestas; lo planificado se fue modificando durante el proceso y en función de las respuestas de estudiantes y sus manifestaciones afectivas, conductuales, cognitivas y agénticas. Se incluyó como herramienta para favorecer la autonomía, el uso de internet y hemerotecas; la visita de profesionales para promover el vínculo entre la teoría y la vida cotidiana, y para sostener la estructura de la clase, se utilizó el feedback formativo como recurso para monitorear y andamiar los avances de estudiantes antes, durante y finalizadas las tareas. 
Finalmente, al terminar el proceso, el estudiantado completó un cuestionario ad hoc y nuevamente completó la escala para valorar cada dimensión de compromiso y el contexto social de la clase. Cabe aclarar que, para el análisis de datos se utilizaron frecuencias porcentuales para describir las respuestas de estudiantes y el coeficiente de correlación Tau-b de Kendall apropiado para estudiar la relación entre dos variables ordinales.

\section{Resultados encontrados}

Los resultados se organizan en torno a tres apartados. En primera instancia, se describe la configuración de la clase en su escenario natural en relación con el estilo docente, especificando las percepciones estudiantiles sobre las dimensiones de autonomía y estructura, así como la asociación entre la configuración percibida y el compromiso reportado en sus cuatro dimensiones. En segunda instancia, se valora, tras la experiencia educativa, nuevamente la asociación entre el estilo docente y el compromiso percibido por el estudiantado. Por último, se analizan las respuestas del alumndo al cuestionario final.

\section{En el aula, ¿cuánto de autonomía y estructura se ofrece?, ¿el estilo docente se vincula con el compromiso del alumnado? Percepciones estudiantiles}

El grupo de estudiantes que participón del estudio ofreció sus percepciones sobre la configuración natural de la clase caracterizando el estilo docente del personal a cargo. Al respecto, y teniendo en cuenta la estructura, manifestaron, como muy cierto, solo un $35 \%$ y $14 \%$, que la maestra se mostraba atenta al grupo de clase sobre la comprensión alcanzada hacia la temática antes de iniciar una nueva y, a la vez, hacia la actividad propuesta, respectivamente. En materia del comportamiento docente predecible, valoraron como muy ciertas las afirmaciones "cada vez que hago algo mal, actúa diferente y cambia la forma de dirigirse", con un $31 \%$ y $22 \%$, correspondientemente. Sobre la comunicación de expectativas, un $18 \%$ declararon como muy cierto que la maestra no dejaba en claro lo que se espera de la actividad, y un $39 \%$ respecto a la clase en general. Finalmente, sobre los apoyos recibidos, mencionaron como muy cierto solo un $20 \%$ ante la afirmación "me enseñan cómo resolver los problemas" y tan solo un $12 \%$ considera también como muy cierto que recibe ayuda de la docente para resolver la actividad, cuando no logra avanzar. 
Contemplando la autonomía, consideran como muy cierto, en un $16 \%$, que la maestra siempre dice lo que hay que hacer $\mathrm{y}$, en el mismo nivel de respuesta, un $33 \%$ valora que la docente se entremete en sus actividades para controlar que se hagan las cosas a su manera. Sobre las alternativas ofrecidas, el $29 \%$ de estudiantes considera como muy cierto que no se ofrecen opciones para hacer las actividades, y un $12 \%$ piensa que se permiten elecciones como una afirmación muy cierta. En relación con la relevancia, únicamente un $12 \%$ aprecia que es muy cierto que la maestra menciona para qué es útil lo que se aprende en el colegio y un $49 \%$ concuerda en que no se explica la importancia de lo enseñado como algo muy cierto. Por último, sobre la dimensión del respeto, un $26 \%$ valora que la maestra escucha sus ideas como muy cierto y un $31 \%$ que eso no sucede.

La configuración de la clase percibida por el estudiantado en función de la valoración del estilo docente muestra direcciones inversas con las dimensiones del compromiso. Específicamente, los resultados evidencian correlaciones lineales negativas entre la dimensión estructura e implicación agéntica $(\mathrm{r}=-.38)$, afectiva $(\mathrm{r}=-.45)$ y conductual $(\mathrm{r}=-.42)$. Ello significa que a medida que el estudiantado más valora las expectativas de trabajo áulico, las respuestas de sus docentes y las ofrecidas son escasas o poco claras, la participación y el interés por las tareas que desarrollan es menor, al igual que las posibilidades de expresar opiniones para alcanzar metas y propósitos propios. La misma tendencia se aprecia entre dicha dimensión del estilo docente y el compromiso cognitivo reportado $(\mathrm{r}=-.32)$, es decir, que la percepción de tener más apoyo por la parte docente se relaciona con un mejor reporte en el uso de estrategias para autorregular los propios procesos de aprendizaje.

Para la dimensión de autonomía, también el compromiso cognitivo reportado se correlaciona negativamente con dicha característica del estilo docente $(r=-.35)$, lo que indica que el estudiantado tiende a percibir que los contextos instructivos que controlan más sus comportamientos y acciones se relacionan con reportes menos ajustados a conductas de autorregulación. También esos contextos que no favorecen tanto la autonomía, el respeto, la relevancia son los que correlacionan con una autoevaluación menor de los componentes afectivos $(\mathrm{r}=.36)$, conductuales $(\mathrm{r}=.39)$ y agénticos $(\mathrm{r}=.42)$ en el compromiso estudiantil. 


\section{Estilo docente mediado por la experiencia educativa, ¿asocia- do a estudiantado comprometido?}

Tras la implementación de la experiencia educativa, el estudiantado encontró, y así se manifiesta en sus respuestas, una configuración de la clase y de las tareas escolares con mayor claridad en estructura $y$, a la vez, con mayores espacios para la autonomía. Sobre la primera dimensión, el $77 \%$ de estudiantes coincidió en que es muy cierto que la maestra monitorea la compresión antes de comenzar una nueva actividad, y en el mismo sentido, un $83 \%$ asegura la comprabación antes de seguir con una clase. En relación con los comportamientos de la maestra, solo en un $15 \%$ y $8 \%$ consideran como muy cierto que la docente se comporta de manera distinta frente a los errores y hacia el grupo, respectivamente. Sobre las expectativas, una proporción muy baja de estudiantes describe como muy cierto que la maestra no las aclara frente a las tareas o lo que se espera de su desempeño en la clase, con un $6 \%$ y $2 \%$, correspondientemente. Por último, un $81 \%$ de estudiantes percibe como muy cierto que los apoyos recibidos muestran cómo resolver un problema, y en un $79 \%$ que el personal docente ayuda a lograrlo de diversas maneras.

Sobre la segunda dimensión, la percepción de control es baja, solo un $10 \%$ y un $2 \%$, identifican como muy cierto que el personal docente dice qué se debe hacer o se entromete en el cuaderno de actividades. No obstante, el respeto es percibido por un $83 \%$ de estudiantes, al mencionar que es muy cierto que la ideas son escuchadas por el personal docente y un $4 \%$ como muy cierto que eso sucede. Asimismo, las posibilidades de elección se perciben como muy ciertas por el $70 \%$ de estudiantes y solo un $10 \%$ valora que no son ofrecidas. Finalmente, un $70 \%$ y $80 \%$ califican como muy cierto que la maestra muestra el qué y cómo se puede usar lo que se aprende en la escuela en la vida cotidiana $\mathrm{y}$, a la vez, por qué es importante saberlo, correspondientemente.

Sobre esta nueva configuración de la clase y las actividades de aprendizaje, el estilo docente muestra tener otra relación con el compromiso autorreportado por el estudiantado. Para ambas dimensiones, se evidencian correlaciones directas y moderadas entre autonomía (ra) y estructura (re) con la dimensión afectiva $(\mathrm{ra}=0,39$; $\mathrm{re}=0,45)$, conductual $(\mathrm{ra}=0,45 ; \mathrm{re}=0,42)$, cognitiva $(\mathrm{ra}=0,46 ; \mathrm{re}=0,48)$ y agéntica (ra $=0,51 ; \mathrm{re}=0,38)$. Es decir, el estudiantado que percibe que los apoyos 
recibidos por sus docentes para desarrollar las tareas de aprendizaje, en términos de feedback formativo, el monitoreo durante la realización de las actividades, y la comunicación de las expectativas, se relacionan con un mayor compromiso autorreportado. Asimismo, son los contextos educativos con docentes que ceden parte del control a sus estudiantes, respetan sus opiniones, ofrecen alternativas y enfatizan la relevancia de lo enseñado, lo que también se relaciona con una mayor participación, interés, y posibilidades de autogestionar los aprendizajes, a la vez, de comunicar maneras o alternativas para moldear el entorno instructivo, en tanto el personal docente se encuentra más abierto a las propuestas de sus estudiantes para implicarse.

\section{Elecciones de estudiantado más comprometido tras la expe- riencia educativa}

De las respuestas al cuestionario final, destacamos que el $94 \%$ de estudiantes estuvieron de acuerdo en que, tras la experiencia educativa, los apoyos orientados a promover la autonomía y una clara estructura de trabajo escolar -en expectativas, seguimientos, retroalimentaciones- se hicieron más presentes en la clase. También, la mayoría (91 \%) reconoce la importancia de que el maestro o maestra posea informaación acerca de sus desempeños con retroalimentaciones orientadas a la autorregulación, al coincidir que les permite avanzar, esforzarse más y mejorar sus aprendizajes, solo una minoría (9\%) considera que tales intercambios sirven fin de obtener una buena nota.

Un aspecto a destacar es que el $25 \%$ aún necesita de apoyos externos para autorregular sus aprendizajes y toma de decisiones; frente a problemas o dificultades, siguen recurriendo en primera instancia a su docente, aunque el $75 \%$ manifiesta releer la consigna y reflexionar sobre lo realizado; asimismo, expresa haber ganado en espacios para expresar sus ideas y proponer forma de hacer, lo que da indicios de la dimensión agéntica del compromiso en el $81 \%$ de estudiantes.

Con respecto a la búsqueda por internet, el $92 \%$ lo tomó como un desafío de nuevos aprendizajes e independencia en saber elegir y tomar decisiones frente a información variada, solo un $8 \%$ lo percibió como dificultad. La misma distribución se vislumbra en la consulta por el apoyo de la visita de personas expertas para definir la utilidad y relevancia de lo aprendido. Finalmente, un $93 \%$ coincide en que las 
consignas fueron claras, comprensibles, flexibles y constructivas, afines a un proceso de aprendizaje sustentado en una buena autonomía y una clara estructura para facilitar la vinculación con las tareas escolares.

En síntesis, las respuestas del estudiantado aluden a las diversas dimensiones que definen el compromiso escolar. En la dimensión agéntica, mencionaron que, durante las clases orquestadas por docentes en el marco de la experiencia educativa, se ofrecieron espacios más abiertos para realizar preguntas, para opinar sobre sus intereses y saberes, así como para ofrecer sugerencias acerca de cómo generar un mejor clima de trabajo áulico a partir de la incorporación de otros recursos o modalidades. En lo que respecta a la dimensión afectiva, expresaron más interés por los contenidos, la forma de aprender y satisfacción por las actividades realizadas en la clase. En relación con la dimensión conductual, quienes participaron de la experiencia expusieron prestar más atención a las explicaciones de su docente, con más participación en los debates y las discusiones que se propusieron en torno a las temáticas trabajadas. Por último, en lo que refiere a la dimensión cognitiva, comentaron la posibilidad de chequear las actividades durante el proceso, planificar cómo las iban a hacer y valorar sus producciones a partir de las retroalimentaciones que su docente ofrecía.

\section{Consideraciones finales}

Partimos de indagar las percepciones de estudiantes sobre la configuración del aula asociada al estilo docente y sus niveles de compromiso hacia las tareas de aprendizaje. Se diseñó una experiencia educativa para andamiar al personal docente en trabajo áulico que promoviera la autonomía y la estructura de la clase, para valorar nuevamente las percepciones sobre ese escenario y su vínculo con la implicación de sus estudiantes.

Los resultados alcanzados muestran que las experiencias educativas basadas en un estilo docente que atiende de manera planificada tanto la estructura como la autonomía, son percibidas como tales por el estudiantado; el cual, a la vez, manifiesta mayor compromiso. Además, revela que apoyar las acciones de estudiantes al contemplar sus intereses, elecciones y retomar la relevancia de las tareas escolares también resulta una pieza clave para la implicación escolar.

Asimismo, la experiencia educativa diseñada y las percepciones construidas antes y después por el estudiantado dan cuenta de la 
condición reflexiva como puente que media entre las valoraciones que construyen como parte de las interacciones que tienen lugar en el aula entre docentes y estudiantes y las tareas escolares. Esa manera particular de mirar y significar la experiencia educativa va dando lugar al cambio de las percepciones sobre ese escenario, pero que a la vez moldea sus maneras de comprometerse. Se desprende de los resultados que la satisfacción de las necesidades psicológicas formuladas desde la $\mathrm{TAD}$, a partir de la modificación del estilo docente contribuye a que el estudiantado se comprometa más.

Esta contribución invita a seguir pensando cómo comprometer, a partir de las experiencias que los sujetos tienen ocasión de experimentar en los entornos instructivos, en tanto entendemos que la experiencia es posible 'por algo', por un acontecimiento externo al estudiantado, tal como moldear el estilo docente, pero que forma para de sus aprendizajes, en tanto el 'algo sucede', algo se mueve, y se traspasa de unas personas a otras y mutuamente ese desplazamiento afecta a estudiantes y docentes (Restrepo, 2014). Por ello, la importancia de aprender a través de la experiencia con el alumnado y utilizarla como base para el desarrollo de la enseñanza.

Los resultados son coincidentes con la investigación desarrollada por Reeve y Lee (2014), quienes encuentran, posicionados desde la $\mathrm{TAD}$, que apoyar la autonomía y ofrecer una adecuada estructura de trabajo áulico, incrementa el compromiso estudiantil. Específicamente, los autores concluyen que el compromiso solo mejora, si en la clase se ofrecen más las oportunidades para satisfacer las necesidades. Aspecto que coincide con un estudio anterior de Jang, Kim y Reeve (2012), quienes hallaron que un maestro o maestra puede generar cambios en la motivación de sus estudiantes a lo largo de los semestres, en tanto se brinden apoyos orientados a sostener la autonomía.

Resaltamos el valor de las implicancias prácticas de la investigación desarrollada y de los resultados encontrados, no solo para comprender la importancia de planificar la clase para lograr los objetivos educativos tendientes a generar estudiantes que se muestren con más vinculación entre sí y con percepciones de mayor interés por participar y generar iniciativas para aprender; sino también apreciar la importancia de reconocer las necesidades del estudiantado en materia de autonomía y competencia, y poder generar diseños instructivos que, tendientes a su promoción, les hagan sujetos activos con manifestaciones afectivas, 
conductuales, cognitivas y agénticas congruentes con mejores aprendizajes y más implicación.

Advertimos la necesidad de seguir indagando a futuro sobre procesos de autorregulación externa e internas (de la Barrera, Rigo y Riccetti, 2019) y su impacto sobre el compromiso cognitivo del estudiantado, en tanto los resultados aún nos indican que el apoyo del personal docente sea clave para autorregular sus aprendizajes, aunque también revelen que la gestión de los propios aprendizajes es mayor cuando logran autosupervisarlo y generar acciones tendientes a modificar el contexto para lograr mayor autonomía en la toma de decisiones.

Por último, asumimos algunas limitaciones que atenderemos en futuros estudios, particularmente, a nivel metodológico, se considera necesario avanzar hacia diseños mixtos, integrando instrumentos que permitan no solo recabar datos cuantitativos, sino también cualitativos, con el uso de narrativas (Contreras y Pérez, 2014), para enriquecer las interpretaciones y la construcción de nuevos conocimientos en materia de experiencia educativa, compromiso y estilo docente.

\section{Referencias}

Albornoz, J., Villablanca, F. y Díaz, A. (2019). El rol del estudiante en el fenómeno del compromiso académico en educación superior. Wimblu, Rev. Estud. de Psic. y Cienc. Soc., 14(1), 81-94. doi: 10.15517/WL.V14I1.35876

Appleton, J. J., Christenson, S. L., Kim, D., y Reschly, A. L. (2006). Measuring cognitive and psychological engagement: Validation of the Student Engagement Instrument. Journal of School Psychology, 44(5), 427-45. https://doi.org/10.1002/pits.20303

Aprender. (2018). Informe Nacional de Resultados. Primaria. $6^{\text {to }}$ año. Recuperado de https://www.argentina.gob.ar/sites/default/files/ aprender2018_primaria.pdf

Arias, M. y Vega, J. (2016). Experiencias integradoras que promueven la autonomía de aprendizajes usando las TIC. Acercando la realidad escolar a las aulas universitarias. Opción, 9, 151-168. Recuperado de https://dialnet.unirioja.es/descarga/articulo/5891160.pdf

Belmont, M., Skinner, E., Wellborn, J. y Connel, J. (1992). Teacher as social context (TASC). Two measures of teacher provision of involvement, structure, and autonomy support. Rochester: University of Rochester. 
CAF. (2018). Políticas para promover la culminación de la educación media en América Latina y el Caribe. Lecciones desde México y Chile. Recuperado de https://www.r4d.org/wp-content/uploads/ CAF-Promoting-Secondary-School-Retention-in-LAC-SPA.pdf Connell, J. P., y Wellborn, J. G. (1991). Competence, autonomy, and relatedness: A motivational analysis of self-system processes. En M. R. Gunnar, y L. A. Sroufe (Eds.), Self-processes and development: The Minnesota symposia on child psychology (pp. 43-77). Hillsdale, NJ: L. Erlbaum Associates.

Contreras, J. y Pérez, N. (2014). Investigar la experiencia educativa. Madrid: Morata.

de la Barrera, M. L., Rigo, D. y Riccetti, A. (2019). La investigación sobre patrones de aprendizaje en Argentina: Conformación de perfiles en estudiantes de educación superior. Revista Colombiana de Educación, 77, 1-31. doi: 10.17227/rce.num77-9521

Deci, E. L., y Ryan, R. M. (2008). Self-determination theory: A macrotheory of human motivation, development and health. Canadian Psychology, 49, 182-185. doi: 10.1037/a0012801

Dubet, F. y Martuccelli, D. (2000). En la escuela. Sociología de la experiencia escolar. Buenos Aires: Losada.

Eccles, J. S., y Midgley, C. (1989). Stage-environment fit: Developmentally appropriate classrooms for young adolescents. En C. Ames, y R. Ames (Eds.), Research on motivation in education: Vol. 3. Goals and cognitions (pp. 13-44). New York: Academic Press.

Eccles, J., Lord, S. y Midgley, C. (1991). What Are We Doing to Early Adolescents? The Impact of Educational Contexts on Early Adolescents. American Journal of Education, 99(4), 521-542. https:// doi.org/10.1086/443996

Fredricks, J., Blumenfeld, P. y Paris, A. (2004). School Engagement: Potential of the Concept, State of the Evidence. Review of Educational Research, 74(1), 59-109.

Fredricks, J., Reschly, A. y Christenson, S. (2019). Interventions for Student Engagement: Overview and State of the Field. En J. Fredricks, A. Reschly, y S. Christenson (Eds.), Handbook of Student Engagement Interventions. Working with Disengaged Students (pp.1-11). London: Academic Press. 
Hospel, V. y Galand, B. (2016). Are both classroom autonomy support and structure equally important for students' engagement? A multilevel analysis. Learning and Instruction, 41, 1-10.

Jang, H., Kim, E. J., y Reeve, J. (2012). Longitudinal test of selfdetermination theory's motivation mediation model in a naturally occurring classroom context. Journal of Educational Psychology, 104, 1175-1188. doi: 10.1037/a0028089

Jang, H., Reeve, J., y Deci, E. L. (2010). Engaging students in learning activities: It is not autonomy support or structure but autonomy support and structure. Journal of Educational Psychology, 102(3), 588-600. http://dx.doi.org/10.1037/a0019682

Jiménez-Jiménez, F., Sosa-Alonso, J., Santana-Bonilla, P. y Hernández-Rivero, V. (2018). La participación del alumnado en el uso de feedback formativo para la mejora de su aprendizaje. En A. Vega Navarro y D. Stendardi (Coords.), De la innovación imaginada a los procesos de cambio (pp. 19-44). Recuperado de https://dialnet.unirioja.es/servlet/articulo? codigo $=6509330$

Könings, K (2007). Student perspectives on education: Implications for instructional redesign. Nederland: Interuniversitair Centrum voor Onderwijsonderzoek. Recuperado de https://www.ou.nl/ documents/40554/111691/proefschrift_Karen_Konings_2007. pdf/6323a9d0-9d87-4e7e-85f1-3a001146af58

Könings, K., Brand-Gruwel, S. y van Merriënboer, J. (2005). Towards more powerful learning environments through combining the perspectives of designers, teachers, and students. British Journal of Educational Psychology, 75, 645-660.

Lam, S., Wong, B., Yang, H. y Liu, Y. (2012). Understanding student engagement with a contextual model. En S. Christenson, A. Reschly y C. Wylie (Eds.), Handbook Research on Student Engagement (pp. 403-419). New York: Springer.

Llorent, V. y Torres-Porras, G. (2018). Innovación docente en el grado de educación primaria. Acercando la realidad escolar a las aulas universitarias. Barcelona: Octaedro.

Mouratidis, A. Michou, A., Aelterman, N., Haerens L. y Vansteenkiste M. (2017). Begin-of-school-year perceived autonomy-support and structure as predictors of end-of-school year study efforts and procrastination: the mediating role of autonomous and controlled 
motivation. Educational Psychology, 38, 435-450. https://doi.or $\mathrm{g} / 10.1080 / 01443410.2017 .1402863$

Oga-Baldwin, W. y Nakata, Y. (2015). Structure also supports autonomy: Measuring anddefining autonomy-supportive teaching in Japanese elementary foreign language classes. Japanese Psychological Research, 57(3), 167-179. https://doi.org/10.1111/ jpr. 12077

Reeve, J. (2013). How Students Create Motivationally Supportive Learning Environments for Themselves: The Concept of Agentic Engagement. Journal of Educational Psychology, 105(3), 579-595.

Reeve, J. (2013). How students create motivationally supportive learning environments for themselves: The concept of agentic engagement. Journal of Educational Psychology, 105, 379-395. https://doi.org/10.1037/a0032690

Reeve, J. (2016). Autonomy-supportive teaching: What it is, how to do it. En J. C. K. Wang, W. C. Liu, y R. M. Ryan's (Eds.), Motivation in educational research: Translating theory into classroom practice (pp. 129-152). New York: Springer.

Reeve, J., y Lee, W. (2014). Students' classroom engagement produces longitudinal changes in classroom motivation. Journal of Educational Psychology, 106, 527-540. https://doi.org/10.1037/ a0034934

Reeve, J., Cheon, S. y Jang, H. R. (2019). A Teacher-Focused Intervention to Enhance Students' Classroom Engagement. En J. Fredricks, A. Reschly, y S. Christenson (Eds.), Handbook of Student Engagement Interventions. Working with Disengaged Students (pp. 87-102). London: Academic Press. https://doi.org/10.1016/ B978-0-12-813413-9.00007-3

Restrepo, A. P. (2014). Investigar las experiencias en lo escolar voces y cuerpos que desde la diversidad interpelan la educación contemporánea. Plumilla Educativa, 14(2), 73-84. Recuperado de https://dialnet.unirioja.es/servlet/articulo? codigo $=5920387$

Rigo, D. (2017). Docentes, tareas y alumnos en la definición del compromiso. Investigando el aula de nivel primario de educación. Educação em Revista, 33, 1-24. http://dx.doi. org/10.1590/0102-4698154275

Rigo, D. y Donolo, D. (2018). Construcción y validación de la Escala de compromiso hacia las tareas escolares en las clases para los 
estudiantes del nivel primario de educación. Revista Psicoespacios, 12(21), 3-22. https://doi.org/10.25057/21452776.1079

Ryan, R. M., y Deci, E. L. (2000). Self-determination theory and the facilitation of intrinsic motivation, social development, and well-being. The American Psychologist, 55, 68-78. https://doi. org/10.1037/0003-066X.55.1.68

Ryan, R. M., y Grolnick, W. S. (1986). Origins and pawns in the classroom: Self-report and projective assessments of individual differences in children's perceptions. Journal of Personality and Social Psychology, 50, 550-558. https://doi. org/10.1037/0022- 3514.50.3.550.

Sampieri, R., Collado, C. y Lucio, P. (2010). Metodología de la investigación ( $5^{\text {ta }}$ ed.). México: Mc Graw Hill.

Santana-Armas, F., Noda-Rodríguez, M. del M. y Pérez-Sánchez, C. (2018). Las experiencias escolares como marco interpretativo de las desigualdades socio-educativas en la educación secundaria: Una aproximación teórica. Forum Sociológico, 32, 29-37. https://doi.org/10.4000/sociologico.1960

Sarrazin, P., Tessier, D., y Trouilloud, D. (2006). Climat motivationnel instauré par l'enseignant et implication des élèves en classe: l'état des recherches. Revue Francaise de Pédagogie, 157, 147177. http://dx.doi.org/10.4000/rfp.463

Schenke, K. (2018). From structure to process: Do students' own construction of their classroom drive their learning? Learning and Individual Differences, 62, 36-48. https://doi.org/10.1016/j. lindif.2018.01.006

Shernoff, D. J., Kelly, S., Tonks, S., Anderson, B., Cavanagh, R., Sinha, S. y Abdi, B. (2016). Student engagement as a function of environmental complexity in high school classrooms. Learning and Instruction, 43, 52-60. Recuperado de https://www.learntechlib. org $/ \mathrm{p} / 199574 /$

Sierens, E., Vansteenkiste, M., Goossens, L., Soenens, B. y Dochy, F. (2009). The synergistic relationship of perceived autonomy-support and structure in the prediction of self-regulated learning. British Journal of Educational Psychology, 79, 57-68. Recuperado de https://www.ncbi.nlm.nih.gov/pubmed/18466671

Stroet, K., Opdenakker, M.-C., y Minnaert, A. (2013). Effects of need supportive teaching on early adolescents' motivation and 
engagement: a review of the literature. Educational Research Review, 9(0), 65-7. http://dx.doi.org/10.1016/j.edurev.2012.11.003

UNESCO (2015). Informe de resultados TERCE: Factores asociados; resumen ejecutivo. Santiago de Chile: OREALC. Recuperado de https://unesdoc.unesco.org/ark:/48223/pf0000243979.

Van Loon, A. Ros, A. y Martens, R. (2012). Motivated learning with digital learning tasks: what about autonomy and structure? Educational Technology Research y Development, 60, 1015-1032.

Veiga, F. (2013). Envolvimento dos alunos na escola: elaboração de uma nova escala de avaliação. International Journal of Developmental and Educational Psychology INFAD Revista de Psicologia , l(1), 441-450.

Zapata, G. y Canet, T. (2008). Propuesta metodológica para la construcción de escalas de medición a partir de una aplicación empírica. Revista Electrónica Actualidades Investigativas en Educación, 8(2), 1-26. Recuperado de http://www.redalyc.org/articulo. oa? $\mathrm{id}=44713044004$ 